\title{
A Direct Imaging Study to Search for and to Characterize Planetary Mass Companions
}

\author{
K. Ward-Duong ${ }^{1 *}$, J. Patience ${ }^{1,2}$, R. J. De Rosa ${ }^{1}$, A. Rajan ${ }^{1}$, \\ P. Hinz ${ }^{3}$, A. Skemer ${ }^{3}$, K. Morzinski ${ }^{3}$, J. Males ${ }^{3}$, L. M. Close ${ }^{3}$, \\ D. W. McCarthy ${ }^{3}$ and C. Kulesa ${ }^{3}$ \\ ${ }^{1}$ School of Earth and Space Exploration, Arizona State University, Tempe, AZ 85287, USA \\ *email: kwardduo@asu.edu \\ ${ }^{2}$ Astrophysics Group, School of Physics, University of Exeter, Exeter EX4 4QL, UK \\ ${ }^{3}$ Steward Observatory, The University of Arizona, Tucson, AZ 85721, USA
}

\begin{abstract}
We present preliminary results from two parallel programs to search for new substellar companions to nearby, young M-stars and to characterize the atmospheres of known planetary mass and temperature substellar companions. For the M-star survey, we are analyzing high angular resolution archival data on systems within 15pc, complementing a subset with well-determined young ages based on measurements of several age indicators. The results include stellar and substellar companion candidates, which we are currently pursuing with follow-up second epoch images. The characterization component of the project involves using LBT LMIRCam and MMT ARIES direct imaging and spectroscopy data to investigate the atmospheres of known young substellar companions with masses overlapping the planetary regime. These atmospheric studies will represent an analogous comparison to the atmospheres of young imaged planets, and provide a means to fundamentally test evolutionary models, enhancing our understanding of the overall substellar population.
\end{abstract}

Keywords. stars: low-mass, stars: binaries, planetary systems, stars: brown dwarfs

\section{M-dwarf Companion Search}

Despite the ubiquity of M-dwarf stars, their population characteristics have not yet been refined in comparison to recent studies of more massive stars (e.g., Raghavan et al. 2010). We present preliminary results from an M-dwarf companion search comprising a volume-limited survey of nearby M-stars within 15 pc, selected using Hipparcos parallaxes and B-V and V-K color criteria. For 188 stars within our sample, we reduced and analyzed available near-infrared, high-resolution data from the Hubble Space Telescope, Canada-France-Hawaii Telescope, and Very Large Telescope archives, searching projected separations of $\sim 1$ to 100 AU from the primary stellar components. The combined data reach median sensitivities to companions with a K-band difference of 9 magnitudes from their primaries at separations of $2^{\prime \prime}$. A total of 19 substellar candidate systems and 30 stellar candidate systems were detected from this survey (two example candidates are shown in the top panel of Figure 1). Follow-up observations, and a comparison with previously published literature measurements, will be carried out to determine the physical association of the detected candidates. From this study, we are deriving the key population statistics of separation distribution, mass ratio distribution, and multiplicity frequency for M-dwarf stars.

\section{Substellar Companion Characterization}

Measurements of the atmospheric compositions of giant extrasolar planets represent an important pathway to understanding the formation and evolution history of exoplanets. 


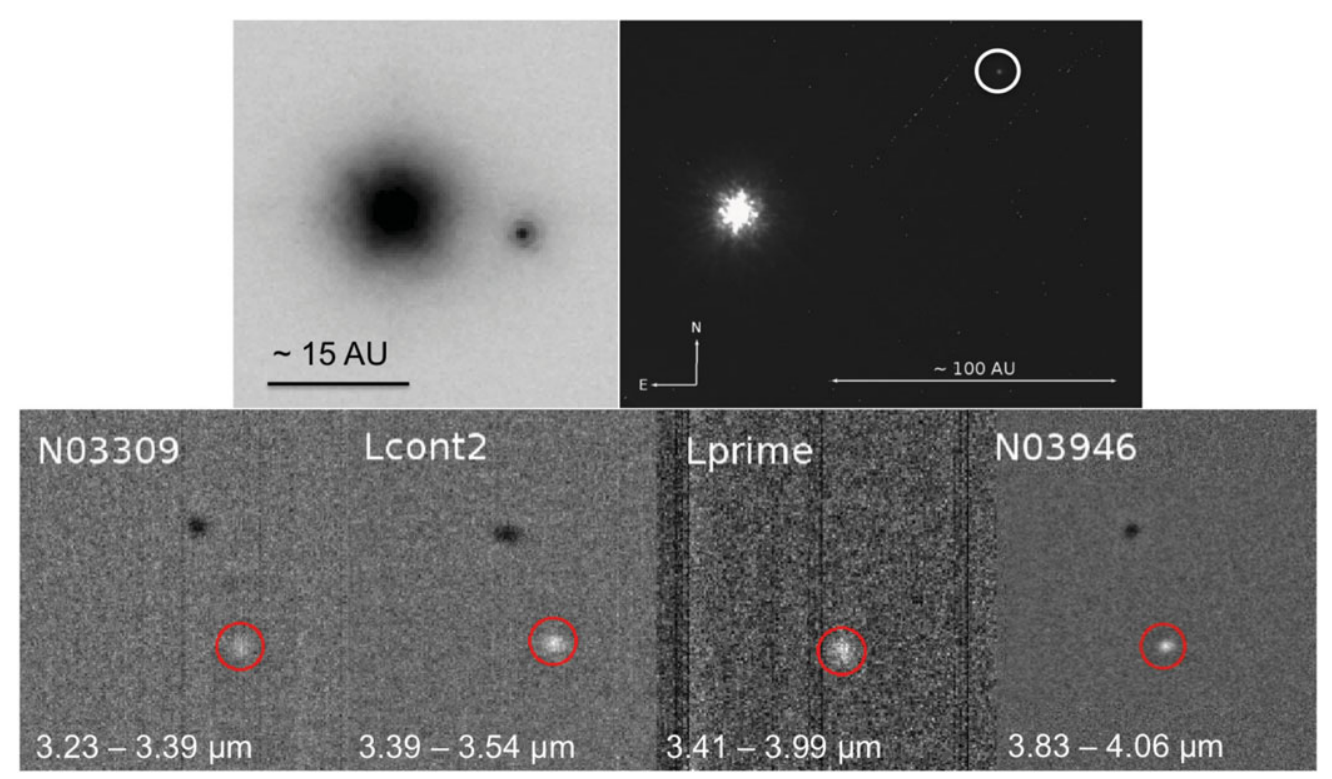

Figure 1. (Top) Examples of two new stellar (left, $\Delta \mathrm{K}$-mag $\sim 4$ ) and substellar (right, $\Delta \mathrm{K}-\mathrm{mag} \sim 7$ ) candidates identified in this survey. (Bottom) LBT narrow and broad L-band difference imaging of G196-3 B, a known substellar companion to an M-dwarf with a projected separation of $\sim 300 \mathrm{AU}$.

Establishing an empirical sequence of object atmospheres spanning the transition between the brown dwarf and planetary regimes provides a critical means of testing these theories and investigating the formation mechanisms of substellar objects. With infrared spectra obtained with the ARIES instrument at the MMT Observatory, L-band imaging from LBT LMIRCam, and visible photometry, we are comparing the spectral energy distributions of substellar objects with theoretical atmospheric models to constrain effective temperatures and bolometric luminosities. These companions exhibit unusually red infrared colors in common with the imaged planetary companions to HR 8799. The young ages of these systems $(\lesssim 400 \mathrm{Myr})$ make them important analogs to directly imaged planets, and allow us to explore the atmospheric properties of low mass, low surface gravity objects. The LBT measurements with different mid-IR filters are particularly sensitive to the effects of clouds, chemistry, and metallicity, which will enable detailed tests of atmospheric models. By combining MMT infrared spectra with the L-band LBT data, we will have comprehensive wavelength coverage of the bulk of the emergent flux from these young substellar objects, allowing us to define an empirical sequence. We are also measuring the $\mathrm{C} / \mathrm{O}$ ratios of young brown dwarf companions to test formation hypotheses by searching for similar or different abundances to the host objects, which may depend on formation via core accretion versus gravitational instability (Öberg et al. 2011). Example LBT thermal IR data on a known substellar companion to an M-dwarf with a projected separation of $\sim 300 \mathrm{AU}$ (G196-3 B) are shown in the bottom panel of Figure 1. Similar studies will be performed on confirmed detections from our M-dwarf survey, and combining our ongoing observations of known companions will allow for atmospheric characterization over a wide range of substellar objects.

\section{References}

Öberg, K. I., Murray-Clay, R., \& Bergin, E. A. 2011, ApJ, 743, 16

Raghavan, D., et al. 2010, ApJS, 190, 1 